\title{
Mitogen-activated protein kinase phosphatase-1 prevents lipopolysaccharide-induced apoptosis in immature rat intestinal epithelial cells
}

\author{
Maria M. Talavera ${ }^{1,2}$, Nathaniel Kralik', Yi Jin', Bernadette Chen ${ }^{1,2}$, Yusen Liu ${ }^{1,2}$ and Leif D. Nelin ${ }^{1,2}$
}

BACKGROUND: Necrotizing enterocolitis is characterized by intestinal inflammation and epithelial barrier dysfunction. Mitogen-activated protein kinase (MAPK) phosphatase (MKP)-1 plays a pivotal role in the feedback control of MAPK signaling, which regulates inflammation and apoptosis. We hypothesized that MKP-1 prevents lipopolysaccharide (LPS)-induced apoptosis in intestinal epithelial cells.

METHODS: Western blot analysis and GPCR were used to assess MKP-1, MAPK (p38, extracellular signal-regulated kinase (ERK), and c-Jun $\mathrm{N}$ terminal kinases (JNK)), caspase 3, caspase 9, tumor necrosis factor (TNF)- $a$, and cyclooxygenase (COX)-2 expression levels in rlEC-6 enterocytes. MKP-1 expression was inhibited using small interfering RNA (siRNA) methodology. Viable cell number was determined using trypan blue exclusion.

RESULTS: LPS stimulation led to activation of p38, JNK, and ERK, and induction of MKP-1 mRNA and protein expression. The induction of MKP-1 was associated with a decrease in p38 phosphorylation, and knockdown of MKP-1 prolonged p38 phosphorylation. While LPS stimulation significantly attenuated proliferation of rIEC-6 cells transfected with scramble siRNA, LPS stimulation resulted in a net decrease in viable cell number in cells transfected with MKP-1 siRNA. Following LPS stimulation, MKP-1 knockdown resulted in greater caspase 3 and 9 activities and greater proinflammatory cytokine (TNF-a, COX-2) expression than in cells transfected with scramble siRNA.

CONCLUSION: Our results demonstrate that MKP-1 has a central role in preventing inflammation-induced apoptosis in rIEC-6 enterocytes.

$\mathbf{N}$ ecrotizing enterocolitis (NEC) is a disease of intestinal inflammation and epithelial barrier dysfunction. This devastating disease affects up to $10 \%$ of premature infants weighing less than $1,500 \mathrm{~g}$ with an overall mortality rate of 25 to $50 \%$ in those requiring surgical intervention (1-3). The primary risk factor is prematurity. Although the exact pathogenesis is not completely understood, an exaggerated inflammatory response to intestinal bacteria has been proposed as a possible contributing mechanism (4). Intestinal epithelial cells (IECs) are the key cell type for the maintenance of intestinal barrier function. They not only provide a strong physical barrier to the external environment but also secrete a variety of antimicrobial factors (5). To maintain the integrity of the intestinal barrier, the intestinal tissue needs to achieve a balance between apoptosis and cell regeneration. This balance is disturbed in the inflamed intestine where the epithelium is exposed to toxins and proinflammatory cytokines that induce apoptosis (6).

IECs also serve a key role in the host immune response to luminal bacteria. Components of the bacteria including lipopolysaccharide (LPS) interact with Toll-like receptors (TLRs) on the IECs themselves. Once activated, TLRs elicit the activation of several signal transduction pathways, including the mitogen-activated protein kinases (MAPK) (7). The MAPK family members can be classified into extracellular signalregulated kinases (ERK), c-Jun $\mathrm{N}$ terminal kinases (JNK), and p38 (ref. 8). While ERKs are preferentially activated by growth factors, JNK and p38 are preferentially activated by stress. MAPKs relay, amplify, and integrate signals to elicit a myriad of cellular responses, such as cell proliferation, differentiation, inflammation, and apoptosis (9).

IECs have a high rate of turnover, thus a balance between proliferation and apoptosis is crucial for maintenance of normal morphology and adequate barrier function of the intestinal wall. Apoptosis may be initiated extrinsically via activation of caspase 8 , or intrinsically via activation of caspase 9. Both pathways lead to caspase 3 cleavage and ultimately result in endonuclease activation and nuclear DNA fragmentation (10). Excessive apoptosis, due to accelerated cell death, leads to the breakdown of the epithelial barrier thus facilitating invasion of pathogenic bacteria. Stress stimuli capable of inducing apoptosis, including LPS, are potent activators of MAPKs. Regulation of MAPK signaling is essential to prevent uncontrolled apoptosis, and one such regulatory mechanism is the MAPK phosphatases (MKPs). The MKPs deactivate ERK, JNK, and p38 by dephosphorylating and thereby terminating stress-induced MAPK 
signaling, and MKP-1 preferentially dephosphorylates p38 and JNK (9,11-15). MKP-1, the archetypical MKP family member, has been shown to regulate numerous physiological and immunological functions (9). For example, MKP-1 has been shown to negatively regulate proliferation in several different types of cells $(16,17)$. The present study was undertaken to test the hypothesis that MKP-1 is central to preventing LPS-induced apoptosis in IECs. We investigated the role of MKP-1 in LPS-induced apoptosis and IEC survival.

\section{RESULTS}

LPS Activates MAP Kinase and MKP-1 Expression in rIEC- 6 Cells MAP kinase activation in confluent rIEC- 6 cells stimulated with $100 \mu \mathrm{g} / \mathrm{ml}$ LPS was evaluated by western blot. LPS treatment resulted in the phosphorylation of p38 (pp38) within $15 \mathrm{~min}$, and levels of pp38 peaked at $30 \mathrm{~min}$ (Figure 1a,b). JNK was phosphorylated at $15 \mathrm{~min}$ after LPS treatment, reaching a peak level at $30 \mathrm{~min}$ before returning to basal levels (Figure 1a,c). ERK peak phosphorylation occurred after 15 min of LPS treatment (Figure 1a,d).

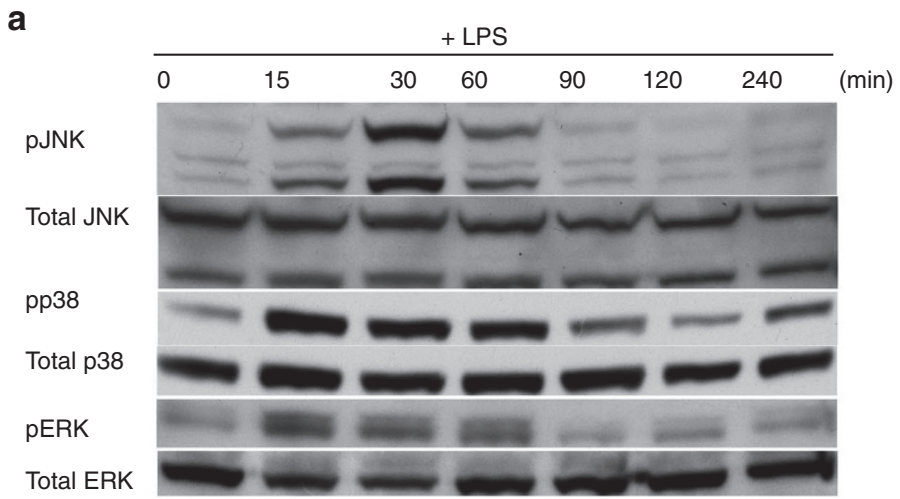

c

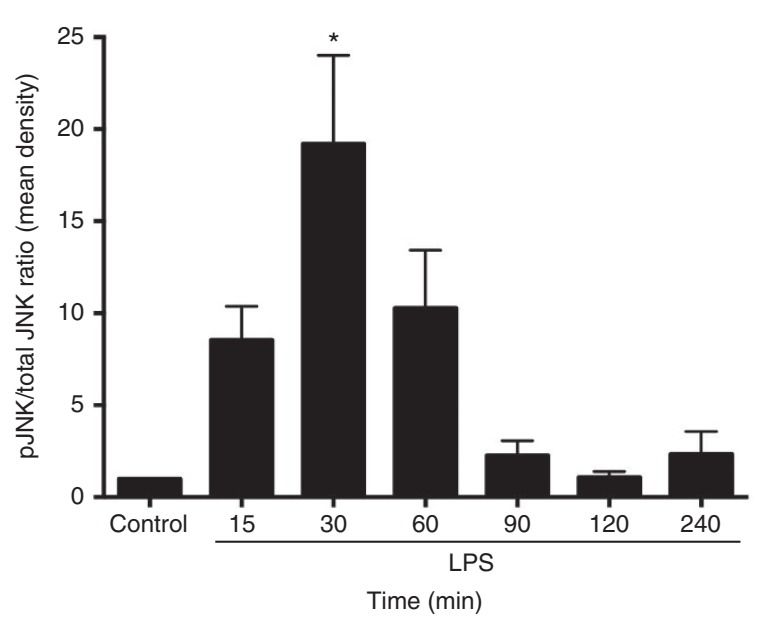

We also examined the temporal expression of MKP-1 in rIEC-6 cells. MKP-1 expression was examined by western blot analysis and qPCR. In unstimulated (control) cells, the MKP-1 protein expression was minimal, essentially below the limit of detection (Figure 2a). In response to LPS stimulation, MKP-1 protein expression increased dramatically, reaching peak levels at $30-60 \mathrm{~min}$ then returning to basal levels by $90 \mathrm{~min}$ (Figure 2b). MKP-1 mRNA levels mirrored protein levels, although MKP-1 mRNA levels returned to below basal levels by $60 \mathrm{~min}$ (Figure 2c). It is worth noting that the increase in MKP-1 protein levels following LPS exposure was temporally associated with a decrease in pp38, phosphorylated ERK (pERK), and phosphorylated JNK (pJNK). These findings suggest that MKP-1 might serve as a negative regulator of MAP kinases in rIEC-6 cells as has been described in leukocytes $(18,19)$.

Temporal Knockdown of MKP-1 Gene Expression in rIEC-6 Cells To characterize the role of MKP-1 in rIEC- 6 cells, rIEC- 6 cells were transfected with either scramble siRNA or MKP-1 interfering siRNA (siMKP-1). Following LPS stimulation of MKP-1 after 30 min, knockdown of MKP-1 mRNA expression was evaluated
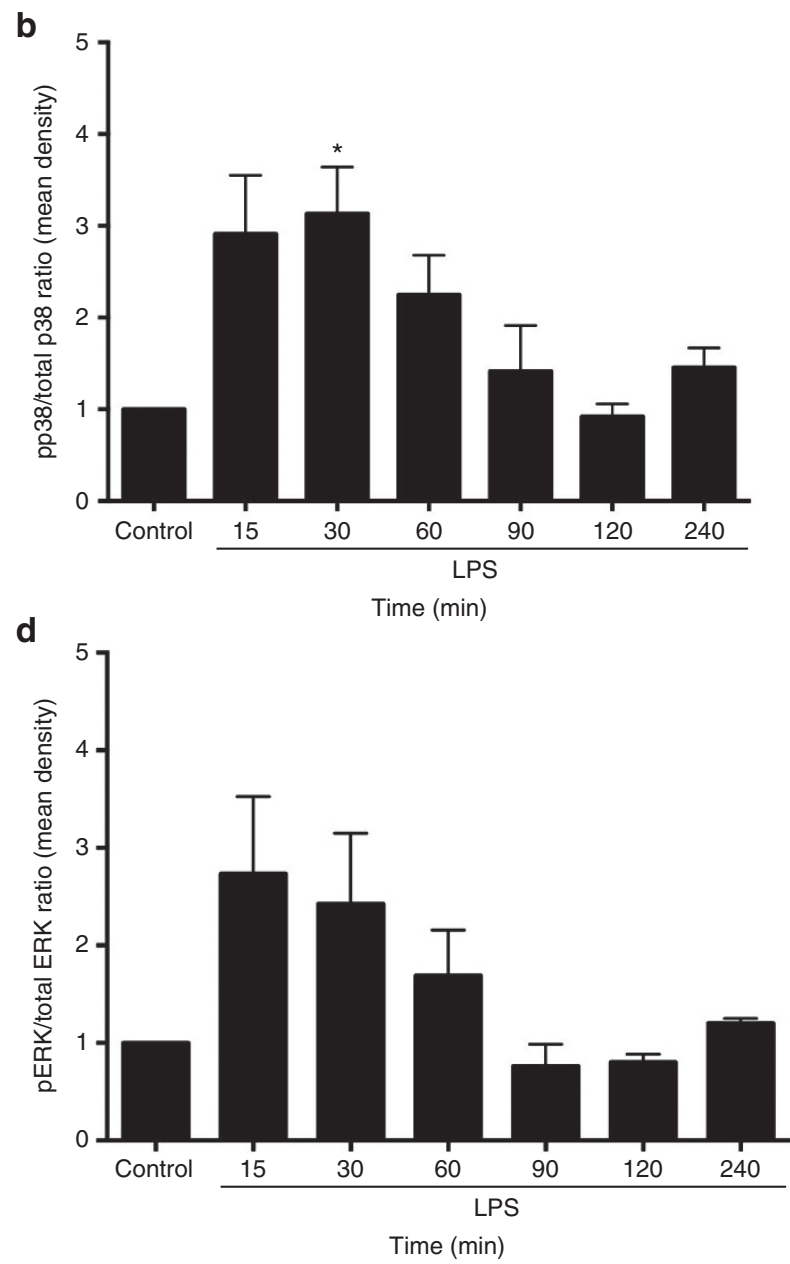

Figure 1. LPS stimulation increased MAP kinase expression in rIEC-6 cells. (a) Representative western blots of phosphorylated and total p38, ERK, and JNK. (b) Densitometry for phospho-p38 (pp38) protein levels normalized to total p38 levels. (c) Densitometry for phospho-JNK (pJNK) levels normalized to total JNK levels. (d) Densitometry for phospho-ERK (pERK) levels normalized to total ERK levels. Data are shown as means \pm SEM relative to each total MAP kinase at each time point from at least three independent experiments with triplicate samples for each time point. *Peak protein expression of pp38 and $\mathrm{pJNK}$ are different from control, $P<0.05$. 
a

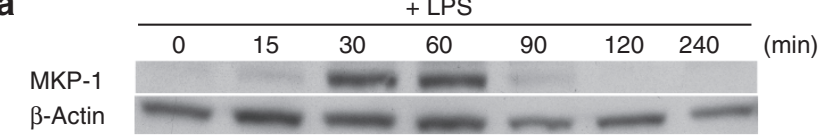

b

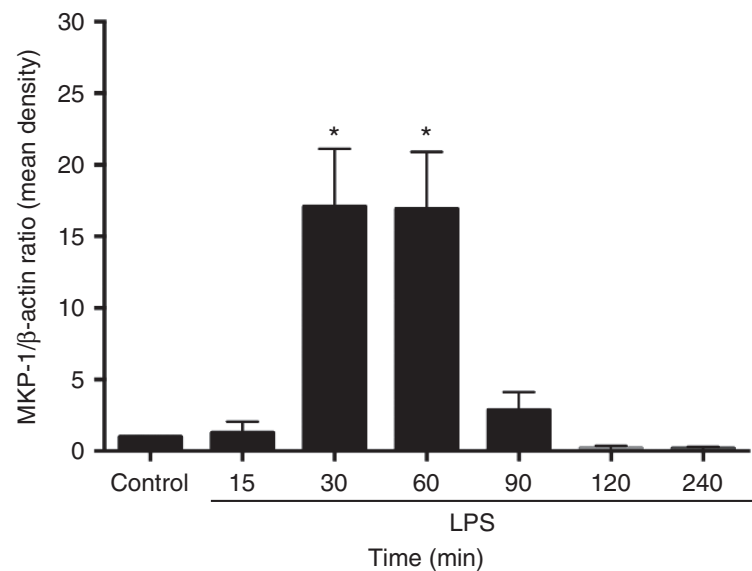

C

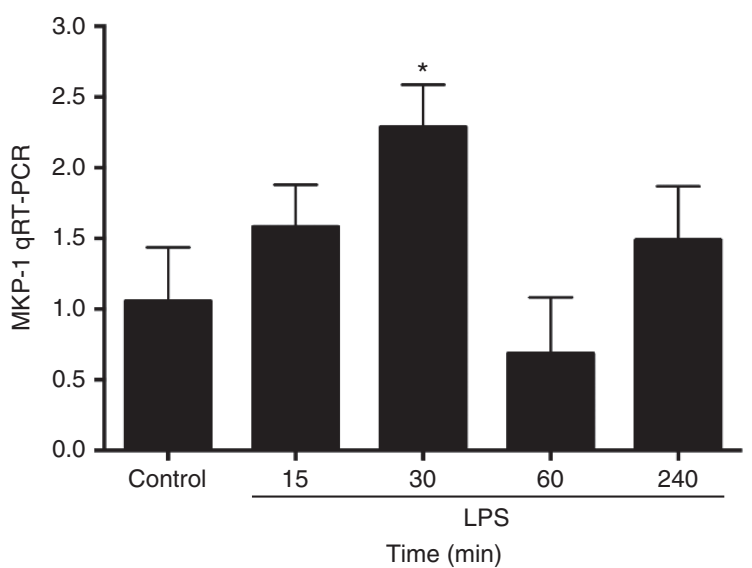

Figure 2. LPS stimulation increased MKP-1 protein expression in rIEC- 6 cells. (a) Representative western blots for MKP- 1 and $\beta$-actin. (b) Densitometry for MKP-1 protein levels normalized to $\beta$-actin protein levels. Data are shown as means \pm SEM relative for MKP-1 protein expression at each time point from at least three independent experiments with triplicate samples for each time point. ${ }^{*} P<0.05$ vs. control. (c) MKP-1 mRNA expression by $\mathrm{qPCR} ;{ }^{*} P<0.05$ vs. control.

at the same time point by qPCR (Figure 3a). Significant attenuation of MKP-1 mRNA expression $(P<0.05)$ was found after $30 \mathrm{~min}$. Decreased MKP-1 mRNA expression was also confirmed at 4 and $24 \mathrm{~h}$ post-LPS stimulation. To evaluate the effect of MKP-1 knockdown on MKP-1 protein expression after prolonged LPS stimulation, MKP-1 protein levels were examined 24 and $48 \mathrm{~h}$ following LPS stimulation. Western blot analyses indicate that MKP-1 protein levels in rIEC-6 cells transfected with siMKP-1 were lower than in cells transfected with scramble siRNA after $48 \mathrm{~h}$ of LPS exposure $(P<0.05$; Figure $3 \mathbf{b}, \mathbf{c})$.

\section{MKP-1 Knockdown Preferentially Prolongs pp38 Activation in rIEC-6 Cells}

To determine the effects of MKP-1 gene knockdown on levels of pp38, pJNK, and pERK, rIEC-6 cells transfected with either scramble or siMKP-1 was examined in a time course experiment. a

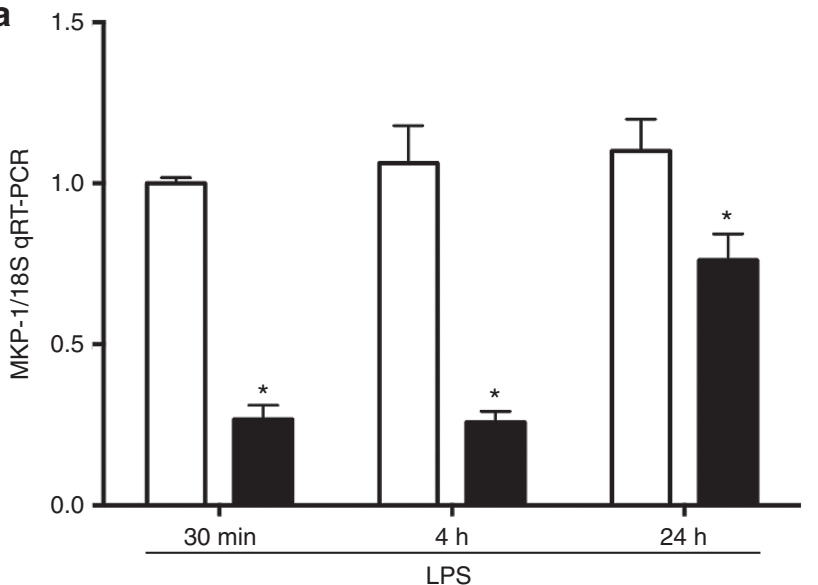

b
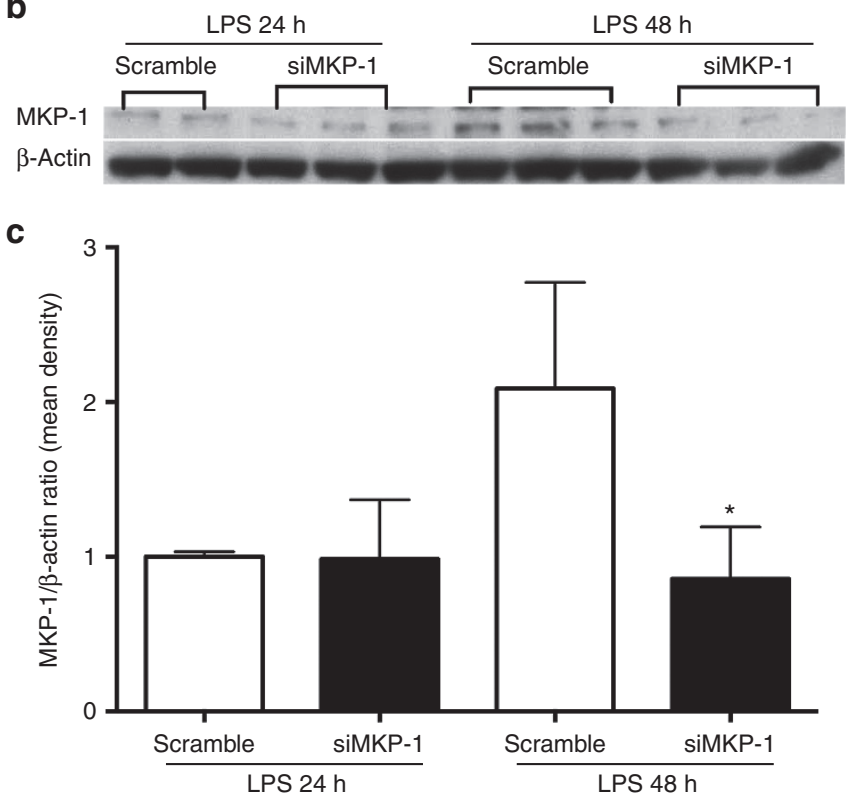

Figure 3. MKP- 1 knockdown in rIEC- 6 cells. (a) rIEC- 6 cells were transiently transfected with either siRNA against MKP-1 (siMKP-1; black bars) or scramble siRNA (scramble; white bars). Twenty-four hours after transfection, the cells were stimulated with LPS $(100 \mu \mathrm{g} / \mathrm{ml})$ for the indicated times. MKP-1 expression was evaluated by qPCR following transfection; ${ }^{*} P<0.05$ vs. scramble for each time point. (b) Representative western blot of MKP-1 protein expression after transfection with siRNA for MKP-1 or scramble siRNA. Following transfection, rlEC cells were stimulated with LPS for 24 and $48 \mathrm{~h}$. (c) Densitometry data shown as means \pm SEM of protein level relative to $\beta$-actin protein levels for triplicate samples from three independent experiments. *Protein expression of MKP-1 after siMKP-1 transfection and LPS stimulation for $48 \mathrm{~h}$ is different from MKP-1 protein expression after scramble transfection and LPS stimulation for $48 \mathrm{~h}, P<0.05$.

Significant upregulation of pp38 $(P<0.05)$ after MKP-1 knockdown was seen following 15,60 , and 90 min of LPS stimulation (Figure 4a,b). In contrast, pERK and pJNK were not significantly affected by MKP-1 knockdown (Figure 4c,d). These findings suggest that MKP-1 is a negative regulator of p38 in rIEC- 6 cells.

\section{MKP-1 Knockdown Augments LPS-induced Expression of TNF- $\alpha$} and COX-2 in rIEC-6 Cells

To investigate the impact of MKP-1 knockdown on the LPSinduced inflammatory response in immature IECs, we first 
a

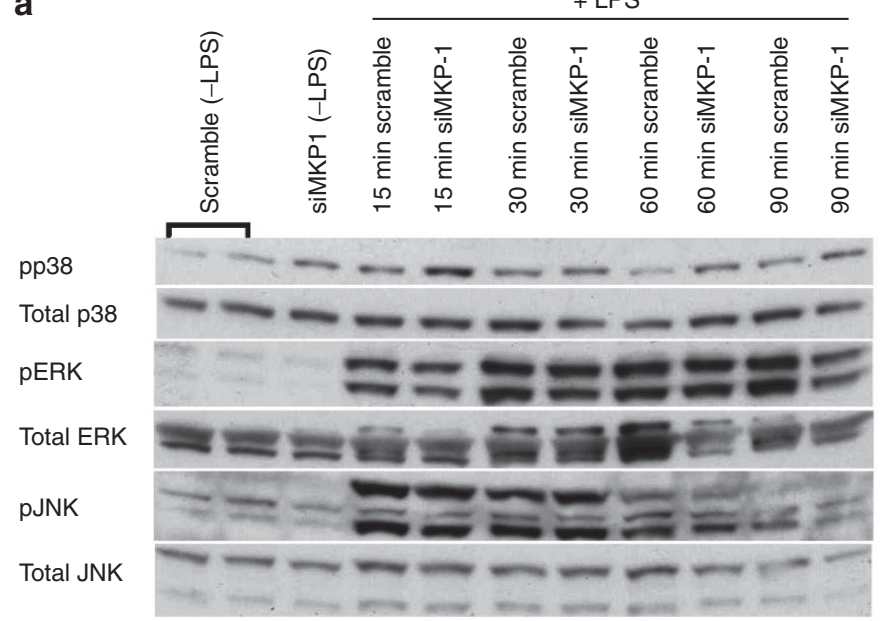

C

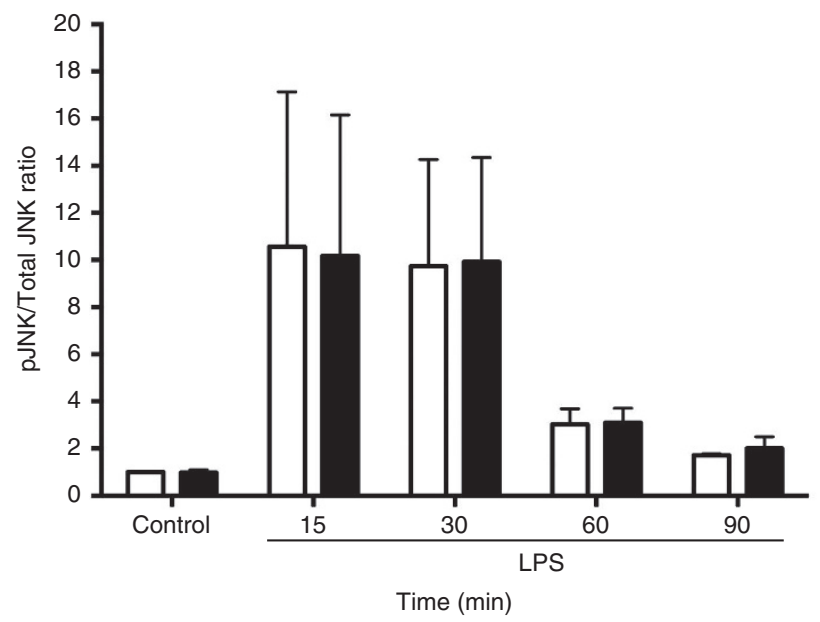

b

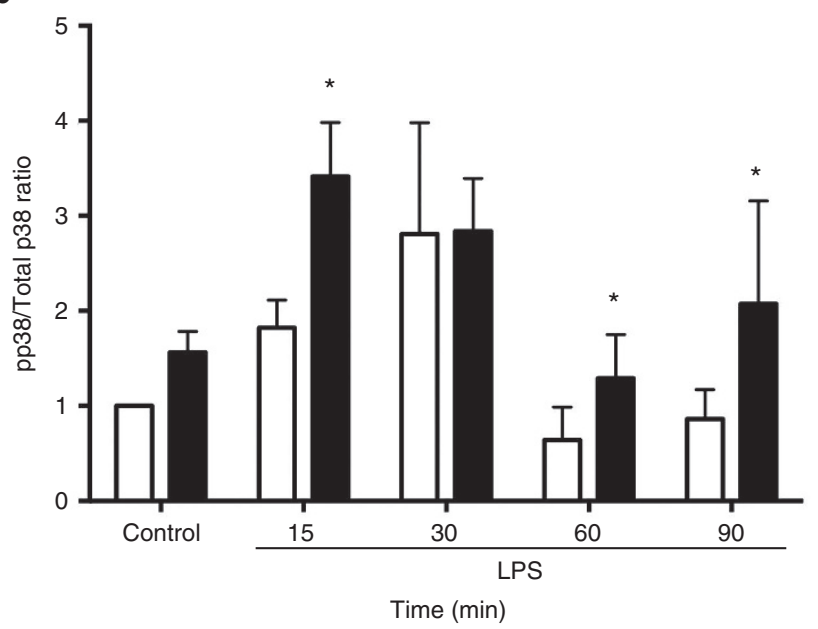

d

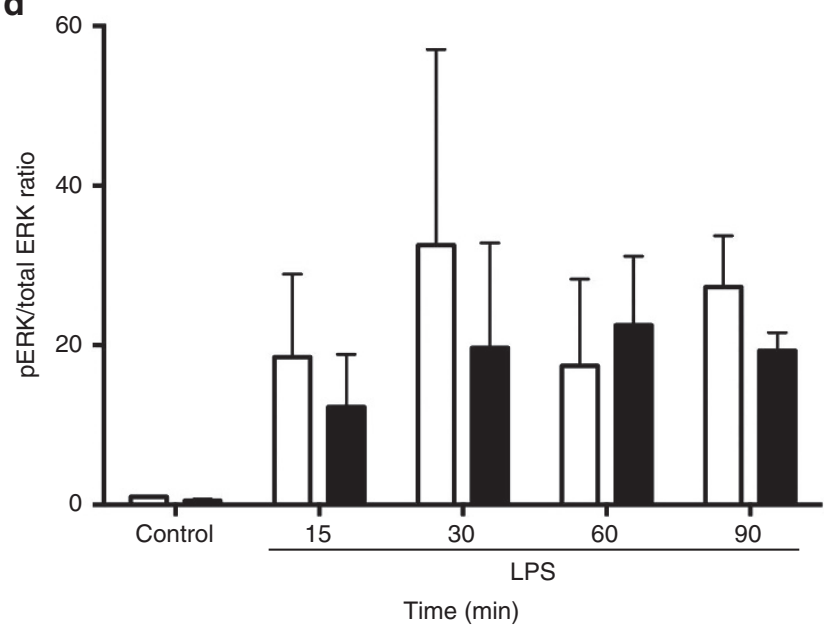

Figure 4. MKP-1 knockdown resulted in more robust and longer lasting pp38 expression in rIEC- 6 cells. (a) rIEC- 6 cells were transiently transfected with either siRNA against MKP-1 (siMKP-1) or scramble siRNA (scramble). Twenty-four hours after transfection, the cells were stimulated with LPS (100 $\mu \mathrm{g} / \mathrm{ml})$ for the indicated times. Control groups were transfected with each transfection vector, respectively, but not stimulated by LPS. Representative western blots of total and phosphorylated p38, ERK, and JNK for each cell lysates are shown. (b) Densitometry for pp38 protein levels normalized to total p38 protein levels. *Peak protein expression of pp38 after siMKP-1 (represented by black bars) transfection was different from scramble (represented by white bars) transfection at 15,60 , and 90 min of LPS stimulation. ${ }^{*} P<0.05$ compared with scramble. (c) Densitometry for pERK protein levels normalized to total ERK protein levels. (d) Densitometry for pJNK protein levels normalized to total JNK protein levels. Data are shown as means \pm SEM relative to total p38, ERK, and JNK at each time point from three independent experiments with triplicate samples for each experimental group.

examined tumor necrosis factor- $\alpha$ (TNF- $\alpha$ ) and cyclooxygenase-2 (COX-2) in rIEC-6 cells following LPS exposure. rIEC-6 cells were grown to $80 \%$ confluence and left unstimulated or stimulated with LPS $(100 \mu \mathrm{g} / \mathrm{ml})$ for $4 \mathrm{~h}$. The cells were then harvested for RNA and analyzed by qPCR. There was increased TNF- $\alpha$ and COX-2 mRNA levels $(P<0.05)$ from LPS stimulated cells as compared with unstimulated IECs (Figure 5a,b).

To test the hypothesis that MKP-1 regulates TNF- $\alpha$ and COX-2 responses in rIEC- 6 cells following LPS stimulation, rIEC-6 cells were transfected with either scramble siRNA or siMKP-1, stimulated with LPS for $4 \mathrm{~h}$, and TNF- $\alpha$ and COX-2 mRNA levels measured by qRT-PCR. Following LPS stimulation, siMKP-1-treated cells produced significantly higher levels of TNF- $\alpha$ and COX-2 mRNA $(P<0.05)$ than did scramble-siRNA transfected cells (Figure $5 \mathrm{c}, \mathrm{d}$ ). Altogether, these findings suggest that MKP-1 plays an important role in the regulation of the inflammatory response in rIEC- 6 cells.

\section{MKP-1 Knockdown Decreases Viable Cell Number}

IECs establish and maintain the intestinal mucosal barrier. To determine the role of MKP-1 in IEC proliferation, cells were transfected with scramble siRNA or siMKP-1 for $24 \mathrm{~h}$ and then seeded at $4 \times 10^{5}$ cells/well in a six-well plate. These cells were then treated with either LPS or vehicle for $48 \mathrm{~h}$ (Figure 6). In the absence of LPS treatment, siMKP-1 transfection of rIEC-6 cells resulted in significant lower numbers of viable cells $(P<$ $0.05)$ than in scramble siRNA-transfected cells. Treatment of scramble-transfected cells with LPS resulted in a 50\% decrease in the number of viable cells as compared with scramble-transfected cells not treated with LPS. Treatment with the siMKP-1 followed by LPS stimulation resulted in a significantly lower number of viable cells than in scramble transfected LPStreated cells $(P<0.0001)$. In fact, the number of viable cells was less than the number of cells that were seeded at the start of the experiment (Figure 6). These findings demonstrate the 
a

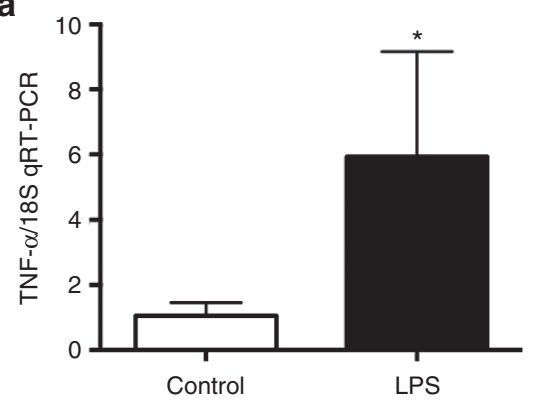

C

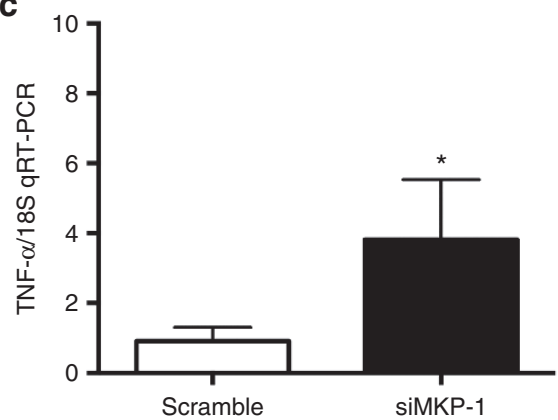

b

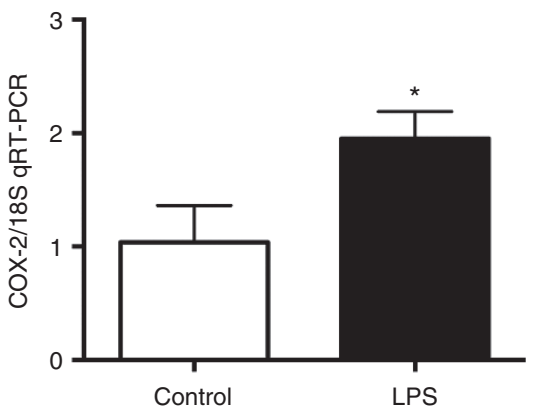

d

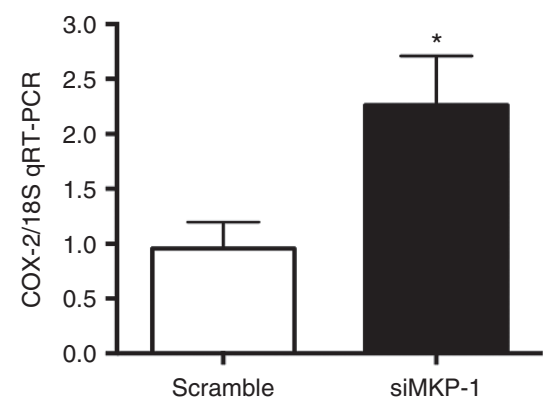

Figure 5. MKP-1 knockdown increased cytokine production in rlEC- 6 cells. rlEC- 6 cells were stimulated with LPS ( $100 \mu \mathrm{g} / \mathrm{ml})$ for $4 \mathrm{~h}$. Control cells remained unstimulated. Proinflammatory mediators, (a) tumor necrosis factor- $\alpha$ (TNF- $\alpha$ ) and (b) cyclooxygenase-2 enzyme (COX-2) mRNA levels were increased post $4 \mathrm{~h}$ LPS stimulation. Data are shown as means \pm SEM of the fold change from control. ${ }^{*} P<0.05$ vs. control. rlEC- 6 cells were transfected with MKP-1 siRNA (siMKP-1) or scramble siRNA (scramble) and stimulated with LPS ( $100 \mu \mathrm{g} / \mathrm{ml}$ ) for $4 \mathrm{~h}$. (c) TNF- $\alpha$ and (d) COX-2 mRNA levels were increased post $4 \mathrm{~h}$ LPS stimulation following indicated transfection. Data are shown as means \pm SEM fold change relative to scramble siRNA-transfected cells. Data shown from three independent experiments done in triplicate; ${ }^{*} P<0.05$ vs. scramble.

critical role of MKP-1 in maintaining rIEC-6 viable cell numbers following LPS stimulation.

\section{MKP-1 Knockdown Increased LPS-induced Apoptosis in rIEC-6 Cells}

To determine the role of MKP-1 in LPS-induced apoptosis of rIEC-6 cells, cells were transfected with either scramble siRNA or siMKP-1. The protein levels of cleaved caspase 9 and caspase 3 were examined by western blot and quantified by densitometry (Figure 7). Following LPS stimulation for 24 or $48 \mathrm{~h}$, cells transfected with siMKP-1 produced significantly higher levels of cleaved caspase $3(P<0.05)$ than did scramble siRNA-transfected cells (Figure $7 \mathbf{b}$ ). Furthermore, after $48 \mathrm{~h}$ of LPS stimulation, cells transfected with siMKP-1 also exhibited significantly higher levels of cleaved caspase $9(P<0.05)$ than did cells transfected with the scramble siRNA (Figure 7c). Taken together, these results demonstrate that MKP-1 plays an important role in dampening the LPS-induced apoptosis response in rIEC-6 cells.

\section{DISCUSSION}

The main findings of this study were: (i) LPS induced a transient activation of MAP kinases, including ERK, JNK, and p38 in rIEC-6 cells, and stimulated MKP-1 expression; (ii) knockdown of MKP-1 led to prolonged activation of p38 following LPS stimulation and resulted in elevated TNF- $\alpha$ and COX-2 expression; (iii) MKP-1 knockdown augmented the LPS-induced decrease in viable cell numbers; and (iv) MKP-1 negatively regulated LPS-induced apoptosis in rIEC-6 cells.
Taken together, these findings strongly support the hypothesis that MKP-1 is central in preventing LPS-induced apoptosis in IECs. These findings show that MKP-1 expression is critical for the maintenance of epithelial cell number. We propose that by inhibiting apoptosis in immature IECs MKP-1 preserves the enterocyte barrier to maintain homeostasis.

Our findings may be particularly relevant to NEC. While the pathogenesis of NEC remains poorly understood, NEC is thought to be a disease of progressive inflammation involving enteric bacteria, the innate immune response, and a compromised intestinal epithelial barrier leading to necrosis (2). MAP kinase activation represents the immediate early signaling events triggered by extracellular stimuli. By regulating the phosphorylation of the downstream targets, MAP kinases transduce the signals from extracellular environment to control the later cellular processes, including proliferation, differentiation, inflammation, and apoptosis. Since MAP kinase activation is an early event that initiates cellular processes that include proliferation and apoptosis, we examined MAP kinase activation shortly after LPS stimulation and assessed later cellular events such as proliferation and apoptosis 24 or $48 \mathrm{~h}$ post stimulation. Because we used cell number as a primary index to quantify cell proliferation, such assay is only meaningful after at least one cell division, underlying the rationale for assessing cell proliferation 24 or $48 \mathrm{~h}$ post-LPS stimulation. Similarly, unlike MAP kinase activation that occurs immediately following extracellular stimulation, apoptosis takes several hours or days to occur (Figure 7). Nonetheless, the critical roles of MAP kinases in apoptosis have been unequivocally established 


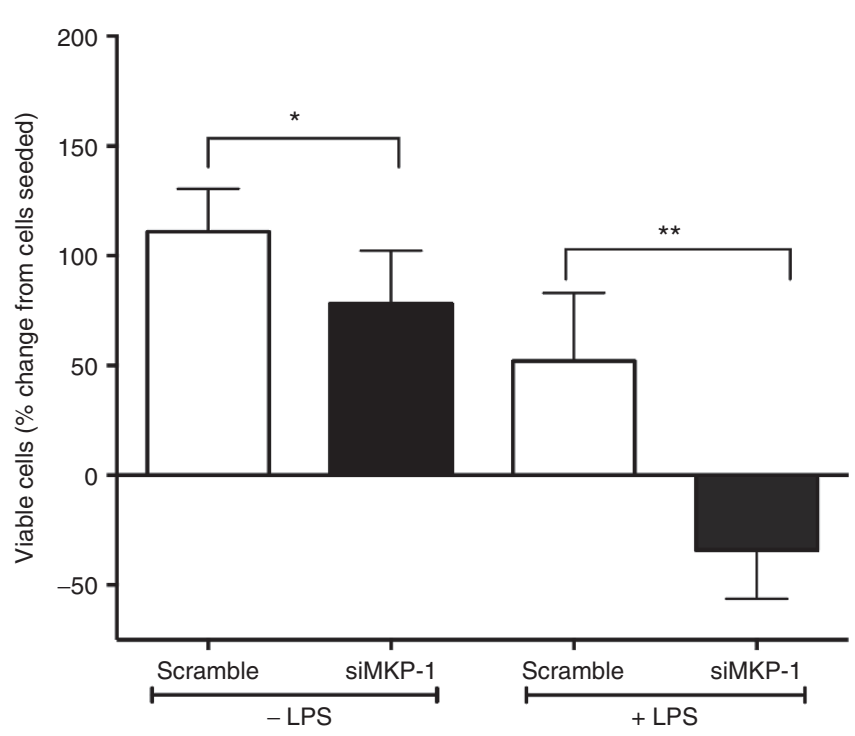

Figure 6. MKP-1 knockdown decreased viable cell number. rIEC-6 cells were transfected with scramble siRNA (scramble) or siRNA against MKP-1 (siMKP-1) and allowed to proliferate for $24 \mathrm{~h}$. Plates were seeded with $4 \times 10^{5}$ cells per well. Cells were then stimulated with high-dose LPS (100 $\mu \mathrm{g} / \mathrm{ml}$ ) for $48 \mathrm{~h}$. Trypan blue exclusion was used to count viable cells. Data are shown as viable cell number as a percent change from cells seeded, with zero being no change in cell number after $48 \mathrm{~h}$ of growth. *siMKP-1, non-LPS-treated cells are different from scramble, non-LPS-treated, $P<$ 0.05. ** siMKP-1, LPS-treated cells are different from scramble, LPS-treated cells, $P<0.0001$. All data shown from three independent experiments done in triplicate. by using constitutive active mutants (20), gene knockout (21), and pharmacological inhibitors (22). The siRNA knockdown experiments presented here clearly established the role of MKP-1 in the regulation of LPS-induced apoptosis in rIEC-6 enterocytes.

MKP-1 may be a therapeutic target for preventing an overzealous inflammatory response that can damage the host, such as is seen in inflammatory bowel disease, NEC, septic shock, etc. (11). MKP-1 deficiency in vivo and in vitro has been found to lead to the excessive production of a wide variety of proinflammatory cytokines (i.e., TNF- $\alpha$ ), chemokines (i.e., MCP1) $(14,19,23)$, as well as iNOS and COX-2 $(13,18,24)$. While previous studies have primarily focused on leukocytes (macrophages, bone marrow-derived dendritic cells, and peritoneal macrophages), the present study sought to uncover the regulatory role of MKP-1 in an immature IEC line during the response to high-dose LPS, as might be seen during the pathogenesis of NEC or inflammatory bowel disease. Interestingly, rIEC-6 cells exhibited a proinflammatory response in terms of TNF- $\alpha$ and COX-2 induction (Figure 5a,b). Knockdown of MKP-1 gene expression enhanced the production of TNF- $\alpha$ and COX- 2 mRNA compared with scramble siRNAtransfected epithelial cells exposed to LPS (Figure 5c,d). This data support the hypothesis that MKP-1 serves as a negative immune regulator in the IEC response to LPS.

Signals from the villus to the crypt cells regulate proliferation, and these signals are mediated by a variety of factors
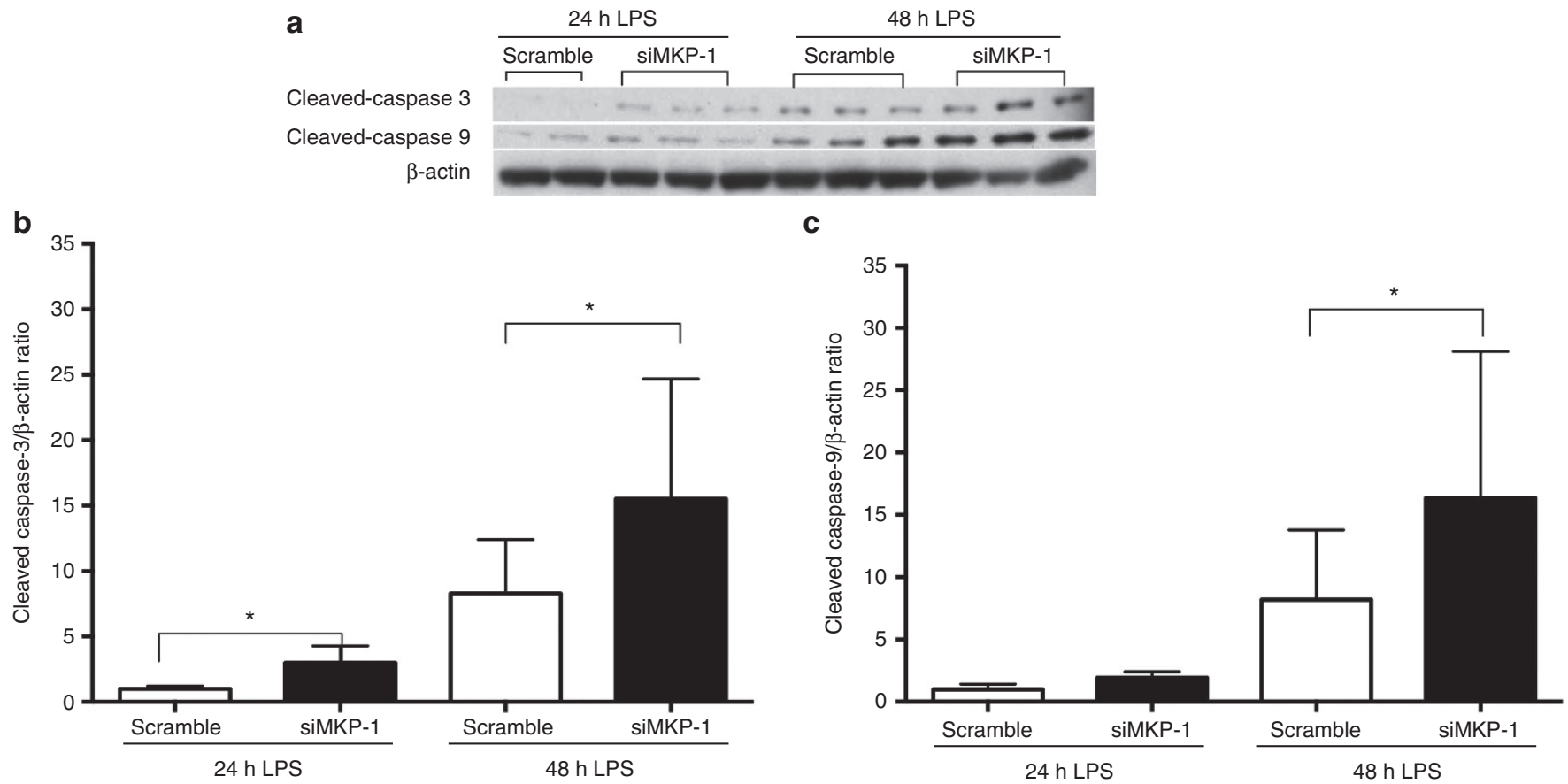

Figure 7. MKP-1 knockdown increased LPS-induced apoptosis in rlEC-6 cells. rlEC-6 cells were transfected with either siRNA against MKP-1 (siMKP-1) or scramble siRNA (scramble). Twenty-four hours after transfection, the cells were stimulated with LPS (100 $\mu \mathrm{g} / \mathrm{ml})$ for 24 or $48 \mathrm{~h}$. Cleaved caspase 3 and cleaved caspase 9 protein expression were analyzed by western blot and normalized to $\beta$-actin. (a) Representative western blots are shown for cleaved caspase 3, cleaved caspase 9, and $\beta$-actin. (b) Densitometry for cleaved caspase-3 and (c) cleaved caspase 9 protein expression normalized to $\beta$-actin. Cleaved caspase 3 protein expression is different between scramble siRNA and siMKP-1 after 24 and 48 h of LPS stimulation ( $\left.{ }^{*} P<0.05\right)$. Cleaved caspase 9 protein expression is different between scramble-transfected cells and siMKP-1 transfected cells only after $48 \mathrm{~h}$ of LPS stimulation $\left({ }^{*} P<0.05\right)$. All data are shown from three independent experiments done in triplicate. 


\section{Articles $\mid$ Talavera et al.}

Table 1. Primers utilized for $\mathrm{qRT}$-PCR

\begin{tabular}{|c|c|c|c|}
\hline Gene & Species & Forward sequence & Reverse sequence \\
\hline TNF- $\alpha$ & Rat & СTACTCCCAGGTTCTCTTCAA & GCAGAGAGGAGGTTGACTTTC \\
\hline COX-2 & Rat & CCACCTCTGCGATGCTCTTC & CATTCCCCACGGTTTTGACATG \\
\hline 18S rRNA & Rat & CCAGAGCGAAAGCATTTGCCAAGA & TCGGCATCGTTTATGGTCGGAACT \\
\hline
\end{tabular}

COX-2, cyclooxygenase-2; MKP-1, mitogen-activated protein kinase phosphatase-1; TNF- $\alpha$, tumor necrosis factor- $\alpha$.

including the MAP kinases (10). The importance of MAP kinase (ERK, JNK, and p38) signaling during IEC proliferation and differentiation has been studied (25-27). By using small molecule inhibition or gene knockout, it has been shown that ERK supports proliferation, whereas p38 and JNK promote differentiation of IECs. Localization of MAP kinases along the crypt-villus axis of the small intestine has been correlated with their biological function (28). Recently, a study has reported on the role of MKP-1 in IEC survival in a different model of apoptotic injury: polyamine depletion following TNF/camptothecin treatment (29). We used a different model of epithelial cell apoptosis, high-dose endotoxin as described in NEC (30). We found that MKP-1 mRNA and protein expression are expressed in undifferentiated, small intestinal epithelial cells (Figure 2) and that MKP-1 is critical to proliferation and viability in these cells (Figure 6). Taken together, these two studies highlight the importance of activated MAP kinase signaling and MKP-1 protein in the regulation of apoptosis and survival of IECs to a various injurious stimuli.

The role of bacterial toxins or endotoxin (LPS) on intestinal barrier function and epithelial cell turnover has been studied (31-33). For example, Ruemmele et al. (31) showed that LPS alone significantly inhibited human IEC proliferation without inducing apoptosis or necrosis. However, we found that prolonged (48h) LPS exposure in conjunction with MKP-1 knockdown triggered increases in both caspase 3 and caspase 9 activities above that found in scramble siRNA-transfected rIEC-6 cells (Figure 7). This is consistent with the observation of decreased cell viability in LPS-treated, scramble siRNAtransfected cells (Figure 6). It should be highlighted that in the absence of MKP-1 knockdown, low degree of apoptosis did occur in rIEC-6 cells in response to prolonged LPS exposure, and knockdown of MKP-1 merely exacerbated LPS-induced apoptosis (Figure $7 \mathrm{~b}, \mathrm{c}$ ). It is likely that the transient activation of p38 triggered a cascade of cellular events including expression of proinflammatory mediators and execution of the apoptotic machinery. In the absence of a functional MKP-1, as the result of the MKP-1 knockdown, p38 activity is substantially augmented and prolonged, exacerbating the inflammatory and apoptotic cascades that aggravates the damage caused by LPS. While MKP- 1 levels in LPS-stimulated cells at $4 \mathrm{~h}$ were dramatically decreased compared with its peak levels (30-60 min), the MKP-1 levels likely remain elevated relative to unstimulated control cells (Figure 2c). Even the residual MKP-1 protein likely plays a role in lowering the basal levels of p38 and antagonizing p38-mediated inflammatory response and growth inhibition. This also explains the growth-inhibitory effects of MKP-1 siRNA in the absence of LPS exposure (Figure 6). If prolonged LPS exposure causes a further suppression of MKP-1 expression, such effect will likely contributes to the adverse outcomes.

While the mechanism by which MKP-1 attenuates apoptosis remains unclear and is beyond the scope of this study, we can offer some speculations. MKP-1 may directly attenuate the signaling pathways activating the apoptosis process. Both JNK and $\mathrm{p} 38$ have been shown to mediate apoptosis in enterocytes $(8,34,35)$. By dephosphorylating and thereby deactivating JNK and p38, MKP-1 may terminate the signal from these pathways, thereby attenuating the signal transduction cascades leading to apoptosis. As MKP-1 knockdown did not significantly alter JNK activity, it is likely that under the study conditions MKP-1 protected rIEC-6 enterocytes largely via deactivation of $\mathrm{p} 38$. Alternatively, MKP-1 may limit the production of factors, such as TNF- $\alpha$, which induce apoptosis in IECs (33). Regardless of the mechanism involved, the novel antiapoptotic function of MKP-1 in this immature intestinal epithelial cell line raises the possibility that MKP-1 may be a potential therapeutic target for the maintenance of IEC viability.

In conclusion, we found that in rIEC-6 enterocytes LPS stimulation upregulated MAP kinase signaling as well as MKP-1 in a counter-regulatory fashion. Most importantly, we have shown using siRNA techniques that MKP-1 is critical to cell viability. These data are consistent with the hypothesis that MKP-1 plays an integral role in the maintenance of the intestinal epithelial barrier by preventing apoptosis. Furthermore, these studies suggest that regulation of MKP-1 may be a therapeutic target for preserving the intestinal epithelial barrier in the setting of overwhelming bacterial inflammation as seen in NEC.

\section{METHODS}

\section{Cell Culture}

Immature murine IECs, rIEC-6, were purchased from the American Type Culture Collection (AManassas, VA). rIEC-6 cells (studied in passages 7-20) are well-described, immortalized, immature, nontransformed rat small intestinal epithelial cells (36). Cells were grown in high glucose Dulbecco's Modified Eagle's medium (Mediatech, Manassas, VA) supplemented with $10 \%$ fetal bovine serum (Hyclone, Salt Lake City, UT), $100 \mathrm{U} / \mathrm{ml}$ penicillin, $100 \mu \mathrm{g} / \mathrm{ml}$ streptomycin, and $0.1 \mathrm{U} / \mathrm{ml}$ of recombinant human insulin. Cells were maintained in $100 \mathrm{~mm}$ tissue culture plates at $37^{\circ} \mathrm{C}$ in a humidified atmosphere with $5 \% \mathrm{CO}_{2}$ in ambient air. Cells were grown to a confluent monolayer prior to experimentation on $60 \mathrm{~mm}$ or six-well plates (Thermo Fisher Scientific, Waltham, MA).

\section{Transfection of MKP-1-siRNA}

rIEC-6 cells were transfected with either a nontargeting control siRNA (scramble siRNA; Integrated DNA Technologies, Coralville, IA) or a MKP-1-specific siRNA (SC-156163; Santa Cruz Biotechnologies, Santa 
Cruz, CA) using a DharmaFECT transfection protocol (Dharmacon RNAi; GE Healthcare, Lafayette, CO). Briefly, rIEC-6 cells were grown in six-well plates up to $\sim 80 \%$ confluence and then incubated with either scramble siRNA or MKP-1 siRNA. Twenty-four hours after transfection, cells were treated with LPS (E. coli 0127:B8; Sigma-Aldrich, St. Louis, MO; $100 \mu \mathrm{g} / \mathrm{ml}$ ) for 24 or $48 \mathrm{~h}$ prior to western blot and qRT-PCR. The LPS dose selected for this study was based on previous studies $(32,37,38)$.

\section{RNA Isolation}

Total RNA was isolated from rIEC-6 cells using Trizol (Invitrogen, Carlsbad, CA), as previously described $(39,40)$. Briefly, $0.7 \mathrm{ml}$ of the Trizol reagent was added to each well of a six-well plate containing rIEC-6 cells and incubated for $5 \mathrm{~min}$ at room temperature. Cells were scraped, and the mixture was collected and chloroform added. The tubes were vortexed for $15 \mathrm{~s}$ and incubated at $30{ }^{\circ} \mathrm{C}$ for $3 \mathrm{~min}$. The mixture was centrifuged at $12,000 \mathrm{~g}$ for $15 \mathrm{~min}$ at $4{ }^{\circ} \mathrm{C}$ before the supernatant was transferred to a fresh $1.5 \mathrm{ml}$ centrifuge tube. Isopropyl alcohol was added, the mixture was incubated at $30^{\circ} \mathrm{C}$ for $10 \mathrm{~min}$, and then centrifuged at $12,000 \mathrm{~g}$ for $10 \mathrm{~min}$ at $4{ }^{\circ} \mathrm{C}$. The supernatant was then discarded. The pellet was washed with $75 \%$ ethanol and centrifuged at $7,500 \mathrm{~g}$ for $5 \mathrm{~min}$ at $4{ }^{\circ} \mathrm{C}$. The supernatant was again discarded; the pellet was partially dried, dissolved in RNase-free water, and stored at $-80^{\circ} \mathrm{C}$.

\section{Reverse Transcription and qPCR}

Reverse transcription was performed as previously described (39). Briefly, $4 \mu \mathrm{g}$ of total RNA was pretreated with RQ1 RNase-free DNase (DNase I endonuclease; Promega, Madison, WI) by incubating at $37^{\circ} \mathrm{C}$ for $30 \mathrm{~min}$ in a total volume of $10 \mu \mathrm{l}$. This reaction was terminated with the addition of RQ1 DNase stop solution. The reaction was then incubated at $65^{\circ} \mathrm{C}$ for $10 \mathrm{~min}$ to inactivate the DNase. The posttreated total RNA then underwent reverse transcription in a total volume of $40 \mu \mathrm{l}$ containing $2.5 \mu \mathrm{mol} / 1 \mathrm{dT}_{16}$ (Applied Biosystems, Foster City, CA), 20 units AMV-RT, $1 \mathrm{mmol} / \mathrm{l}$ dNTP, $1 \times$ AMV-RT buffer (Promega), and RNase-free water. The samples were incubated in a PCR-iCycler (Bio-Rad, Hercules, CA) at $42{ }^{\circ} \mathrm{C}$ for $60 \mathrm{mins}, 95{ }^{\circ} \mathrm{C}$ for $5 \mathrm{~min}$, and stored at $-20^{\circ} \mathrm{C}$. Quantitative real-time PCR was performed with the Chromo 4 Real-time PCR Detection System (Bio-Rad), using qPCR SYBR Green Master-mix (Thermo Fisher Scientific). PCR reactions were performed for 40 cycles using the following parameters: $95^{\circ} \mathrm{C}$ for $15 \mathrm{~s}, 55^{\circ} \mathrm{C}$ for $30 \mathrm{~s}$, and $72{ }^{\circ} \mathrm{C}$ for $30 \mathrm{~s}$. The melting curves were manually verified for the presence of a single product. For each reaction, negative controls containing reaction mixture and primers without cDNA were performed to verify that primers and reactions mixtures were free of template contamination. The primers for each gene are listed in Table 1 and each was normalized to $18 \mathrm{~S}$ rRNA using the $\Delta \Delta \mathrm{Ct}$ method (41). All samples were analyzed in triplicate.

\section{Protein Isolation}

Protein was isolated from rIEC- 6 cell lysate as previously described $(40,42)$. Briefly, rIEC-6 cells were washed with ice-cold phosphatebuffered saline (PBS), and $50-100 \mu \mathrm{l}$ of lysis solution $(0.2 \mathrm{~mol} / \mathrm{l}$ $\mathrm{NaOH}, 0.2 \%$ sodium dodecyl sulfate) was added to each plate or each well of a six-well plate. Thirty minutes before use, the following protease inhibitors were added to each $\mathrm{ml}$ of lysis solution: $1 \mu \mathrm{l}$ aprotinin $\left(10 \mathrm{mg} / \mathrm{ml}\right.$ in double distilled (dd) $\left.\mathrm{H}_{2} \mathrm{O}\right), 1 \mu \mathrm{l}$ leupeptin $(10 \mathrm{mg} / \mathrm{ml}$ in $\left.\mathrm{ddH}_{2} \mathrm{O}\right)$, and $1 \mu \mathrm{l}$ of phenylmethylsulfonyl fluoride $(34.8 \mathrm{mg} / \mathrm{ml}$ methanol). The rIEC-6 cells were scraped, collected into sterile centrifuge tubes, and placed on ice for $30 \mathrm{~min}$. The cell lysates were centrifuged at $12,000 \mathrm{~g}$ for $15 \mathrm{~min}$. The supernatant was stored at $-80^{\circ} \mathrm{C}$. Total protein concentration was determined by the Bradford method (43) using a commercially available assay kit (Bio-Rad).

\section{Western Blot}

The lysed rIEC- 6 cells were assayed for total and phosphorylated p38, JNK, and ERK, as well as MKP-1, cleaved caspase 3, and cleaved caspase 9 protein levels as previously described $(39,40)$. Aliquots of cell lysate were diluted 1:1 with sodium dodecyl sulfate sample buffer, heated to $80^{\circ} \mathrm{C}$ for $15 \mathrm{~min}$, and then centrifuged at $10,000 \mathrm{~g}$ at room temperature for $2 \mathrm{~min}$. Aliquots of supernatant were used for sodium dodecyl sulfate-polyacrylamide gel electrophoresis. The proteins were transferred to polyvinylidene difluoride membranes and blocked for $2 \mathrm{~h}$ in PBS with $0.1 \%$ Tween (PBS-T) containing 5\% nonfat dried milk. The membranes were then incubated overnight at $4{ }^{\circ} \mathrm{C}$ with one of the following primary antibodies: MKP-1 rabbit polyclonal antibody (Santa Cruz); phosphorylated ERK, p38 and JNK rabbit polyclonal antibodies, total p38 and JNK antibodies, and cleaved caspase 3 and cleaved caspase 9 antibodies (Cell Signaling Technology, Beverly MA); and total ERK antibody (Transduction Laboratories, Lexington, $\mathrm{KY})$. After overnight incubation, the membranes were washed three times with PBS-T. The membranes were then incubated with horseradish peroxidase-conjugated goat antirabbit or goat antimouse IgG secondary antibody (Bio-Rad) at room temperature for $1 \mathrm{~h}$ and subsequently washed three times with PBS-T. The protein bands were visualized using enhanced chemiluminescence plus reagent (GE Health Sciences, Piscataway, NJ) and quantified using densitometry (Sigma Gel; Jandel Scientific, San Rafael, CA). To control for protein loading, the blots were stripped using a stripping buffer containing $62.5 \mathrm{mmol} / \mathrm{l}$ Tris $\mathrm{HCl}$ (pH 6.8), $2 \%$ sodium dodecyl sulfate, and 100 $\mathrm{mmol} / \mathrm{l} \beta$-mercaptoethanol, and the blots were reprobed for $\beta$-actin (1:10,000; Abcam, Cambridge, MA) as described above.

\section{Proliferation Assay}

To determine cell proliferation, rIEC- 6 cells were seeded in six-well plates at a density of $4 \times 10^{5}$ cells/well in Dulbecco's Modified Eagle's media as described above and incubated for $48 \mathrm{~h}$. Adherent cells were trypinsized, and viable cells were counted using trypan blue exclusion. To determine endotoxin effect alone on rIEC-6 proliferation, adherent rIEC-6 cells were incubated with LPS (E. coli 0127:B8) for $48 \mathrm{~h}$ prior to trypan blue exclusion counting. To determine the effect of MKP-1 knockdown on rIEC- 6 cell proliferation and cell viability, adherent cells were transfected with either scramble siRNA or MKP-1 siRNA for $24 \mathrm{~h}$ prior to seeding into six-well plates $\left(4 \times 10^{5}\right.$ cells/well). The effects of LPS exposure to the proliferation of scramble and siMKP1-transfected rIEC-6 cells were determined after incubating the cells with LPS (E. coli 0127:B8) for $48 \mathrm{~h}$ prior to counting viable cells.

\section{Statistical Analysis}

Values are given as means \pm SEM. All experiments were done in triplicate and conducted in three independent experiments to verify results. T-test or one-way ANOVA was used to compare groups. Differences were considered significant when $P<0.05$ (Prism; GraphPad Software, San Diego, CA).

\section{STATEMENT OF FINANCIAL SUPPORT}

No financial assistance was received to support this study.

Disclosure:There are no financial disclosures or conflicts of interest to report.

\section{REFERENCES}

1. Lin PW, Stoll BJ. Necrotising enterocolitis. Lancet 2006;368:1271-83.

2. Neu J, Walker WA. Necrotizing enterocolitis. N Engl J Med 2011;364: $255-64$.

3. Sharma R, Hudak ML. A clinical perspective of necrotizing enterocolitis: past, present, and future. Clin Perinatol 2013;40:27-51.

4. Claud EC, Walker WA. Hypothesis: inappropriate colonization of the premature intestine can cause neonatal necrotizing enterocolitis. FASEB J 2001;15:1398-403.

5. McCracken VJ, Lorenz RG. The gastrointestinal ecosystem: a precarious alliance among epithelium, immunity and microbiota. Cell Microbiol 2001;3:1-11.

6. Schulzke JD, Bojarski C, Zeissig S, Heller F, Gitter AH, Fromm M. Disrupted barrier function through epithelial cell apoptosis. Ann NY Acad Sci 2006;1072:288-99.

7. De Plaen IG. Inflammatory signaling in necrotizing enterocolitis. Clin Perinatol 2013;40:109-24.

8. Ray RM, Jin S, Bavaria MN, Johnson LR. Regulation of JNK activity in the apoptotic response of intestinal epithelial cells. Am J Physiol Gastrointest Liver Physiol 2011;300:G761-70.

9. Liu Y, Shepherd EG, Nelin LD. MAPK phosphatases-regulating the immune response. Nat Rev Immunol 2007;7:202-12.

10. Ramachandran A, Madesh M, Balasubramanian KA. Apoptosis in the intestinal epithelium: its relevance in normal and pathophysiological conditions. J Gastroenterol Hepatol 2000;15:109-20. 
11. Li L, Chen SF, Liu Y. MAP kinase phosphatase-1, a critical negative regulator of the innate immune response. Int J Clin Exp Med 2009;2:48-67.

12. Chi H, Barry SP, Roth RJ, et al. Dynamic regulation of pro- and antiinflammatory cytokines by MAPK phosphatase 1 (MKP-1) in innate immune responses. Proc Natl Acad Sci USA 2006;103:2274-9.

13. Hammer M, Mages J, Dietrich H, et al. Dual specificity phosphatase 1 (DUSP1) regulates a subset of LPS-induced genes and protects mice from lethal endotoxin shock. J Exp Med 2006;203:15-20.

14. Zhao Q, Wang X, Nelin LD, et al. MAP kinase phosphatase 1 controls innate immune responses and suppresses endotoxic shock. J Exp Med 2006;203:131-40.

15. Wancket LM, Frazier WJ, Liu Y. Mitogen-activated protein kinase phosphatase (MKP)-1 in immunology, physiology, and disease. Life Sci 2012;90:237-48.

16. Comalada M, Lloberas J, Celada A. MKP-1: a critical phosphatase in the biology of macrophages controlling the switch between proliferation and activation. Eur J Immunol 2012;42:1938-48.

17. Liao Q, Guo J, Kleeff J, et al. Down-regulation of the dual-specificity phosphatase MKP-1 suppresses tumorigenicity of pancreatic cancer cells. Gastroenterology 2003;124:1830-45.

18. Wang X, Meng X, Kuhlman JR, et al. Knockout of Mkp-1 enhances the host inflammatory responses to gram-positive bacteria. J Immunol 2007;178:5312-20.

19. Shepherd EG, Zhao Q, Welty SE, Hansen TN, Smith CV, Liu Y. The function of mitogen-activated protein kinase phosphatase- 1 in peptidoglycanstimulated macrophages. J Biol Chem 2004;279:54023-31.

20. Xia Z, Dickens M, Raingeaud J, Davis RJ, Greenberg ME. Opposing effects of ERK and JNK-p38 MAP kinases on apoptosis. Science 1995;270:1326-31.

21. Emhemmed F, Ali Azouaou S, Thuaud F, et al. Selective anticancer effects of a synthetic flavagline on human Oct4-expressing cancer stem-like cells via a p38 MAPK-dependent caspase-3-dependent pathway. Biochem Pharmacol 2014;89:185-96.

22. McLean LS, Crane L, Baziard-Mouysset G, Edwards LP. Antiproliferative effect induced by novel imidazoline S43126 in PC12 cells is mediated by ROS, stress activated MAPKs and caspases. Pharmacol Rep 2014;66:937-45.

23. Chen P, Li J, Barnes J, Kokkonen GC, Lee JC, Liu Y. Restraint of proinflammatory cytokine biosynthesis by mitogen-activated protein kinase phosphatase-1 in lipopolysaccharide-stimulated macrophages. J Immunol 2002;169:6408-16.

24. Lasa M, Abraham SM, Boucheron C, Saklatvala J, Clark AR. Dexamethasone causes sustained expression of mitogen-activated protein kinase (MAPK) phosphatase 1 and phosphatase-mediated inhibition of MAPK p38. Mol Cell Biol 2002;22:7802-11.

25. Aliaga JC, Deschênes C, Beaulieu JF, Calvo EL, Rivard N. Requirement of the MAP kinase cascade for cell cycle progression and differentiation of human intestinal cells. Am J Physiol 1999;277(3 Pt 1):G631-41.

26. Houde M, Laprise P, Jean D, Blais M, Asselin C, Rivard N. Intestinal epithelial cell differentiation involves activation of $\mathrm{p} 38$ mitogen-activated protein kinase that regulates the homeobox transcription factor CDX2. J Biol Chem 2001;276:21885-94.

27. Tong C, Yin Z, Song Z, et al. c-Jun NH2-terminal kinase 1 plays a critical role in intestinal homeostasis and tumor suppression. Am J Pathol 2007;171:297-303.
28. Fu Z, Kim J, Vidrich A, Sturgill TW, Cohn SM. Intestinal cell kinase, a MAP kinase-related kinase, regulates proliferation and G1 cell cycle progression of intestinal epithelial cells. Am J Physiol Gastrointest Liver Physiol 2009;297:G632-40.

29. Bavaria MN, Jin S, Ray RM, Johnson LR. The mechanism by which MEK/ ERK regulates JNK and p38 activity in polyamine depleted IEC-6 cells during apoptosis. Apoptosis 2014;19:467-79.

30. Hunter CJ, De Plaen IG. Inflammatory signaling in NEC: role of $\mathrm{NF}-\kappa \mathrm{B}$, cytokines and other inflammatory mediators. Pathophysiology 2014;21:55-65.

31. Ruemmele FM, Beaulieu JF, Dionne S, et al. Lipopolysaccharide modulation of normal enterocyte turnover by toll-like receptors is mediated by endogenously produced tumour necrosis factor alpha. Gut 2002; $51: 842-8$.

32. Yu LC, Flynn AN, Turner JR, Buret AG. SGLT-1-mediated glucose uptake protects intestinal epithelial cells against LPS-induced apoptosis and barrier defects: a novel cellular rescue mechanism? FASEB J 2005;19:1822-35.

33. Ruemmele FM, Dionne S, Levy E, Seidman EG. TNFalpha-induced IEC-6 cell apoptosis requires activation of ICE caspases whereas complete inhibition of the caspase cascade leads to necrotic cell death. Biochem Biophys Res Commun 1999;260:159-66.

34. Franklin CC, Kraft AS. Conditional expression of the mitogen-activated protein kinase (MAPK) phosphatase MKP-1 preferentially inhibits $\mathrm{p} 38$ MAPK and stress-activated protein kinase in U937 cells. J Biol Chem 1997;272:16917-23.

35. Franklin CC, Srikanth S, Kraft AS. Conditional expression of mitogenactivated protein kinase phosphatase-1, MKP-1, is cytoprotective against UV-induced apoptosis. Proc Natl Acad Sci USA 1998;95:3014-9.

36. Quaroni A, Wands J, Trelstad RL, Isselbacher KJ. Epithelioid cell cultures from rat small intestine. Characterization by morphologic and immunologic criteria. J Cell Biol 1979;80:248-65.

37. Yamada T, Inui A, Hayashi N, Fujimura M, Fujimiya M. Serotonin stimulates endotoxin translocation via 5-HT3 receptors in the rat ileum. Am J Physiol Gastrointest Liver Physiol 2003;284:G782-8.

38. Imaeda H, Yamamoto H, Takaki A, Fujimiya M. In vivo response of neutrophils and epithelial cells to lipopolysaccharide injected into the monkey ileum. Histochem Cell Biol 2002;118:381-8.

39. Chicoine LG, Paffett ML, Young TL, Nelin LD. Arginase inhibition increases nitric oxide production in bovine pulmonary arterial endothelial cells. Am J Physiol Lung Cell Mol Physiol 2004;287:L60-8.

40. Nelin LD, Nash HE, Chicoine LG. Cytokine treatment increases arginine metabolism and uptake in bovine pulmonary arterial endothelial cells. Am J Physiol Lung Cell Mol Physiol 2001;281:L1232-9.

41. Livak KJ, Schmittgen TD. Analysis of relative gene expression data using real-time quantitative PCR and the 2(-Delta Delta C(T)) Method. Methods 2001;25:402-8.

42. Jin Y, Calvert TJ, Chen B, et al. Mice deficient in Mkp-1 develop more severe pulmonary hypertension and greater lung protein levels of arginase in response to chronic hypoxia. Am J Physiol Heart Circ Physiol 2010;298:H1518-28.

43. Bradford MM. A rapid and sensitive method for the quantitation of microgram quantities of protein utilizing the principle of protein-dye binding. Anal Biochem 1976;72:248-54. 